\title{
Hinweise für Autoren/Sonderbände
}

Bitte senden Sie Ihr Manuskript direkt an den Verlag S. Karger.

Das Manuskript soil in druckreifem Zustand in dreifacher Ausfertigung eingereicht werden. Die Entscheidung über die Aufnahme erfolgt aufgrund mehrerer Gutachten. Eine Kopie des Manuskripts soil grundsätzlich beim Autor verbleiben.

Die typographische Gestaltung und redaktionelle Bearbeitung ist dem

Verlag vorbehalten.

Autorkorrekturen über 10\% der Satzkosten hat der Autor zu tragen.

Dem Autor stehen 40 Gratissonderdrucke seiner Arbeit zu.

Verlagsrecht. Mit der Annahme des Manuskriptes gehen alle Rechte an Sonder- und

Nachdrucken, an Abdrucken in anderen Publikatio-nen sowie an Ubersetzungen auf den Verlag über. Ebenso erhält der Verlag das Recht von Vervielfältigungen (auch von Abbildungen) jeder Art wie auch Vortrag, Funk, Fernsehen sowie Speicherung in Datenverarbeitungsanlagen - auch von Teilen eines Manuskripts.

Markennamen müssen, wenn sie geschützt sind, mit einem hochge-stellten ${ }^{\circledR}$ bezeichnet werden. Die Verantwortung für die korrekte Befolgung dieser Vorschrift liegt ausschließlich beim Autor. Abkürzungen sollen möglichst sparsam verwendet werden. Bei spe-ziellen Abkürzungen sollte der Autor jeweils beim ersten Auftreten das Wort ausschreiben und die später verwendete Abkürzung in Klammern folgen lassen: z.B. Adenosin-Monophosphat (AMP).

Institutsangabe in der Sprache der Arbeit.

Zusammenfassungen sollen 20 Schreibmaschinenzeilen nicht über-schreiten. Eine Fassung in englischer Sprache und der englische Titel der Arbeit müssen mitgeliefert werden, auch wenn die Arbeit in deutscher Sprache abgefaßt ist. In die Zusammenfassung gehören keine Literaturzitate, Tabellen, Abbildungen und Abbildungshinweise sowie allgemein gehaltene Aussagen.

Schlồsselwörter sollen ebenfalls in deutscher und englischer Sprache abgefaßt werden. Zu einer Arbeit gehören drei bis fünf Key Words

Tabellen, Abbildungen und Literaturzitate müssen im Text erwähnt werden.

Abbildungsvorlagen dürfen nicht aufgeklebt, gelocht oder mit Büro-klammern angeheftet eingereicht werden und sollen auf der Rückseite den Namen des Autors, den Titel des Beitrags sowie die Bildnummer tragen.

Adresse des Autors ist am Schíuß der Arbeit vollständig anzugeben.

Literaturverzeichnis. Es soil nur die im Text zitierten Publikationen, diese dafür lückenlos, enthalten. Die Literatur ist entweder alphabe-tisch zu ordnen oder entsprechend der Reihenfolge im Text zu nume-rieren.

Sonderhefte/Sonderbände 
Sonderhefte bzw. Sonderbände sind nur in beschränkter Auflage vorrätig. Der Preis ist abhängig vom Umfang. Abonnenten erhalten diese zu einem um 20 \% vom Ladenpreis ermäßigten Vorzugspreis.

Beiträge zur Infusionstherapie und klinischen Ernährung

Sonderheft Nr. 1: Dextran-Symposium Krems 197360 Seiten, DM16,-

Sonderheft Nr. 2: II. Internationales Symposium Erlangen 1973-

Bilanzierte Ernährung in der Therapie

96 Seiten, DM 20-

Sonderheft Nr. 3: Chemisch definierte Diät

36 Seiten, DM15,-

Sonderband 1: Parenterale Ernährung

Deutsch-Skandinavisches Symposium, Kopenhagen, Juni 1978

VI, 286 Seiten, DM 30 -

Sonderband 2: Infusionstherapie bei Volumenmangel und bei rheologischen Indikationen

Symposium auf Schloß Korb, März 1978 VI, 122 Seiten, 63 Abb., 27 Tab., DM20,-

Sonderband 3: Mikrofiltration und andere Transfusionsprobleme

in der Intensivmedizin

Symposium in Hamburg, März 1978

VI, 86 Seiten,.30 Abb., 26 Tab., DM 20-

Sonderband 4: Richtlinien zur Infusionstherapie mit Aminosäuren

Symposium in Mainz, Juli 1978

VI, 102 Seiten, 29 Abb., 25 Tab., DM 20-

Sonderband 5: Systematisierung von Infusionslösungen und

Grundlagen der Infusionstherapie

VIII, 107 Seiten, 6 Abb. , 32 Tab. , 1980, DM 25-

Sonderband 6: Leukozytenseparation und -transfusion

VIII, 192 Seiten, 25 Abb., 43 Tab.. 1981, DM60,-

Sonderband 7: Ernährung und Gesundheit

Eine Untersuchung bei jungen Frauen und Männern in Heidelberg

XII, 204 Seiten, 77 Abb., 41 Tab., 1981, DM 40-

Sonderband 8: Infusionstherapie mit Dextranen

Symposium in Krems, Mai 1980

VI, 114Seiten,20Abb.,26Tab., 1981, DM25,-

Sonderband 9: 7S-Immunglobulin zur intravenösen Anwendung -

Theorie und Klinik

Symposium in Heidelberg, Mai 1982

VIII, 175 Seiten, 53 Abb., 35 Tab., 1982, DM 60-

Sonderband 10: Sepsis unter besonderer Berücksichtigung der Ernäh-rungsprobleme. VIII +208 S. , 68 Abb. , 46 Tab. , 1983, DM 60-

Sonderband 11: Immunglobulintherapie - Grundlagen und klinische Anwendung. VIII, 96

Seiten, 28 Abb., 25 Tab., 1983, DM 30 -

Sonderband 12: Pflanzenfasern - Neue Wege in der Stoffwechsel-therapie. Guar-

SymposiumMünchen, 1983 VIII, 224 Seiten, 81 Abb., 65 Tab., 1983, DM 65-

Beiträge zur Intensiv- und Notfallmedizin (neu bei Karger!) 
Band 1: Organversagen in der Intensivmedizin - Akutes Lungen-versagen, akutes

Leberversagen, Sepsis und septischer Schock XI, 182 S., 61 Abb. (davon 6 farbig), 67 Tab., geb., DM 75-

Band 2: Kritische Bewertung aktueller Therapiemaßnahmen

in der Intensivmedizin 